\title{
FUZZY IMPLICATIVE FILTERS OF BE-ALGEBRAS BASED ON THE THEORY OF FALLING SHADOWS
}

\author{
Ravi Kumar Bandaru, N. Rafi and Bijan Davvaz
}

\begin{abstract}
Based on the idea of falling shadows and fuzzy sets, the notion of a falling fuzzy implicative filter of a BE-algebra is introduced. Relations between fuzzy implicative filters and falling fuzzy implicative filters are provided.
\end{abstract}

Keywords: falling shadow, fuzzy implicative filter, falling fuzzy implicative filter.

\section{Introduction}

In the study of a unified treatment of uncertainty modelled by mixing likelihood and fuzzy pure mathematics, Goodman[3] discovered the equivalence between a fuzzy set and a category of random sets. Wang and Sanchez[12] introduced the idea of falling shadows that directly relates likelihood ideas with the membership perform of fuzzy sets. The falling shadow illustration theory shows us the manner of choice relaid on the joint degrees distribution. It is an affordable and convenient approach for the theoretical development and, therefore, the sensible applications of fuzzy sets and fuzzy logics. The mathematical structure of the idea of falling shadows is developed in[11]. Tan et al. [9, 10] established a theoretical approach to outline a fuzzy inference relation, and fuzzy set operations supported the idea of falling shadows. Jun and Park[5] mentioned the notion of a falling fuzzy subalgebra/ideal of a BCK/BCI-algebra. Jun et al.[4] additionally thought of falling fuzzy positive implicative ideals. They introduced the notion of a falling fuzzy positive implicative ideal of a BCK-algebra, supported the idea of falling shadow and provided relations between falling fuzzy positive implicative ideals and falling fuzzy ideals. In [13] Zhan et al. introduced the notions of falling fuzzy (implicative)filters of $R_{0}$ algebras and supported the idea of falling shadows and fuzzy sets. They provided relations between fuzzy (implicative) filters and falling fuzzy (implicative) filters, and applied the idea of falling fuzzy inference relations to $R_{0}$ algebras and obtained some connected results. In [1] Bandaru et al. mentioned the notion of a

Received June 21, 2016; accepted August 06, 2016

2010 Mathematics Subject Classification. Primary 06F35, 03E72; Secondary 03G25 
falling fuzzy filter of a BE-algebra.

In this paper, we tend to use the idea of falling shadows to ascertain a falling fuzzy implicative filter in an exceedingly BE-algebra as a generalization of a fuzzy implicative filters in BE-algebras. We offer a relation between fuzzy implicative filters and falling fuzzy implicative filters. We tend to show that each falling fuzzy implicative filter could be a $T_{m}$-fuzzy implicative filter.

\section{Preliminaries}

Definition 2.1. [6] An algebra $(X, *, 1)$ of type $(2,0)$ is called a BE-algebra if for all $x, y, z \in X$ the following identities hold:

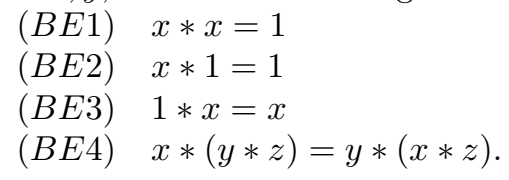

We introduce a binary relation $\leq$ on $X$ by $x \leq y$ if and only if $x * y=1$. It is easy to see that for any $x \in X$,

$$
1 \leq x \Rightarrow x=1 .
$$

Proposition 2.1. [7] If $(X, *, 1)$ is a BE-algebra then $x *(y * x)=1$ for any $x, y \in X$.

Definition 2.2. [7] A BE-algebra $(X, *, 1)$ is said to be transitive if for all $x, y, z \in$ $X$, it satisfies $y * z \leq(x * y) *(x * z)$.

Definition 2.3. [6] A filter of a BE-algebra $X$ is a subset $F$ of $X$ such that for all $x, y \in X$ :

$(F 1) 1 \in F$

$(F 2)$ if $x * y \in F$ and $x \in F$, then $y \in F$.

We will denote by $\operatorname{Fil}(X)$ the set of all filters in a BE-algebra $X$. It is easy to see that $\{1\}, X \in \operatorname{Fil}(X)$.

Example 2.1. [7] Consider the set $X=\{1, a, b, c, d\}$ with the following table of $*$ :

\begin{tabular}{|c|c|c|c|c|c|}
\hline$*$ & 1 & $\mathrm{a}$ & $\mathrm{b}$ & $\mathrm{c}$ & $\mathrm{d}$ \\
\hline 1 & 1 & $\mathrm{a}$ & $\mathrm{b}$ & $\mathrm{c}$ & $\mathrm{d}$ \\
\hline $\mathrm{a}$ & 1 & 1 & $\mathrm{~b}$ & $\mathrm{c}$ & $\mathrm{b}$ \\
\hline $\mathrm{b}$ & 1 & $\mathrm{a}$ & 1 & $\mathrm{~b}$ & $\mathrm{a}$ \\
\hline $\mathrm{c}$ & 1 & $\mathrm{a}$ & 1 & 1 & $\mathrm{a}$ \\
\hline $\mathrm{d}$ & 1 & 1 & 1 & $\mathrm{~b}$ & 1 \\
\hline
\end{tabular}

Then $(X, *, 1)$ is a BE-algebra and $\operatorname{Fil}(X)=\{\{1\},\{1, a\},\{1, b\},\{1, b, c\},\{1, a, b, d\}, X\}$. 
Definition 2.4. [7] A BE-algebra $(X, *, 1)$ is said to be self-distributive if $x *(y *$ $z)=(x * y) *(x * z)$ for all $x, y, z \in X$.

Example 2.2. [8] Consider the set $X=\{1, a, b, c, d\}$ with the following table of $*$ :

\begin{tabular}{|c|c|c|c|c|c|}
\hline$*$ & 1 & $\mathrm{a}$ & $\mathrm{b}$ & $\mathrm{c}$ & $\mathrm{d}$ \\
\hline 1 & 1 & $\mathrm{a}$ & $\mathrm{b}$ & $\mathrm{c}$ & $\mathrm{d}$ \\
\hline $\mathrm{a}$ & 1 & 1 & $\mathrm{~b}$ & $\mathrm{c}$ & $\mathrm{d}$ \\
\hline $\mathrm{b}$ & 1 & $\mathrm{a}$ & 1 & $\mathrm{c}$ & $\mathrm{c}$ \\
\hline $\mathrm{c}$ & 1 & 1 & $\mathrm{~b}$ & 1 & $\mathrm{~b}$ \\
\hline $\mathrm{d}$ & 1 & 1 & 1 & 1 & 1 \\
\hline
\end{tabular}

Then $X$ is a BE-algebra satisfying self-distributivity.

Proposition 2.2. [8] Let $(X, *, 1)$ be a self-distributive BE-algebra. Then for any $x, y, z \in X$,

(i) if $x \leq y$ then $z * x \leq z * y$ and $y * z \leq x * z$;

(ii) $y * z \leq(z * x) *(y * x)$.

Every filter $F$ of a BE-algebra $X$ has the following assertion:

for any $x, y \in X$, if $x \leq y$ and $x \in F$, then $y \in F$.

Definition 2.5. [7] A non-empty subset $F$ of a BE-algebra $X$ is called an implicative filter of $X$, if it satisfies $(F 1)$ and

$$
(\forall x, y, z \in X)(x *(y * z) \in I, x * y \in I \Rightarrow x * z \in I)
$$

Definition 2.6. [2] A fuzzy set $\mu$ in a BE-algebra $X$ is called a fuzzy filter of $X$ if it satisfies for all $x, y \in X$ :

$(G 1) \mu(1) \geq \mu(x)$

(G2) $\mu(y) \geq \min \{\mu(x * y), \mu(x)\}$

Lemma 2.1. [7] Let $\mu$ be a fuzzy filter of a BE-algebra X. Then the following conditions hold for all $x, y \in X$.

(i) $\mu(x * y)=\mu(1)$ implies $\mu(x) \leq \mu(y)$

(ii) $x \leq y$ implies $\mu(x) \leq \mu(y)$.

Theorem 2.1. [7] A fuzzy set $\mu$ of a BE-algebra $X$ is a fuzzy filter in $X$ if and only if it satisfies the following conditions:

(i) $\mu(1) \geq \mu(x)$ for all $x \in X$

(ii) $\mu(x * z) \geq \min \{\mu(x *(y * z), \mu(y))\}$ for all $x, y, z \in X$.

Definition 2.7. [8] Let $\mu$ be a fuzzy set in a BE-algebra $X$. For any $\alpha \in[0,1]$, the set $\mu_{\alpha}=\{x \in X \mid \mu(x) \geq \alpha\}$ is called a level subset of $\mu$. 
Proposition 2.3. [2] Let $\mu$ be a fuzzy set in a BE-algebra X. Then $\mu$ is a fuzzy filter of $X$ if and only if its nonempty level subset $\mu_{\alpha}=\{x \in X \mid \mu(x) \geq \alpha\}$ is a filter of $X$ for all $\alpha \in[0,1]$.

Corollary 2.1. [2] If $\mu$ is a fuzzy filter of a BE-algebra $X$ then the set

$$
X_{b}=\{x \in X \mid \mu(x) \geq \mu(b)\}
$$

is a filter of $X$ for every $b \in X$.

Corollary 2.2. [2] If $\mu$ is a fuzzy filter of a BE-algebra $X$ then the set

$$
X_{\mu}=\{x \in X \mid \mu(x)=\mu(1)\}
$$

is a filter of $X$.

Definition 2.8. [7] A fuzzy set $\mu$ in a BE-algebra $X$ is called a fuzzy implicative filter of $X$ if it satisfies (G1) and

$$
(\forall x, y, z \in X)(\mu(x * z) \geq \min \{\mu(x *(y * z)), \mu(x * y)\})
$$

Note that each fuzzy implicative filter could be a fuzzy filter, however, the converse is not true. In fact, we have a tendency to contemplate a BE-algebra $X=\{1, a, b, c, d\}$ with a Cayley table that is given in Example 2.1. Let $\mu$ be a fuzzy set in $X$ outlined by $\mu(1)=\mu(a)=0.9$ and $\mu(b)=\mu(c)=\mu(d)=0.2$. Then $\mu$ could be a fuzzy filter of $X$, however, it is not a fuzzy implicative filter of $X$ since $\mu(b * c) \nsupseteq$ $\min \{\mu(b *(d * c)), \mu(b * d)\}$.

Proposition 2.4. [7] Let $\mu$ be a fuzzy set in a BE-algebra $X$. Then $\mu$ is a fuzzy implicative filter of $X$ if and only if its nonempty level subset $\mu_{\alpha}$ is an implicative filter of $X$ for all $\alpha \in[0,1]$.

Proposition 2.5. [7] Let $\mu$ and $\nu$ be two fuzzy filters of a transitive BE-algebra $X$ with $\mu \leq \nu$ and $\mu(1)=\nu(1)$. If $\mu$ is a fuzzy implicative filter of $X$, then so is $\nu$.

We will now show the fundamental theory on falling shadows. We refer the reader to the papers $[3,9,10,11,12]$ for any information concerning the idea of falling shadows.

Given a universe of discourse $U$, let $\mathfrak{P}(U)$ denote the power set of $U$. For each $u \in U$, let

$$
\dot{u}:=\{E \mid u \in E \text { and } E \subseteq U\}
$$

and for each $E \in \mathfrak{P}(U)$ let

$$
\dot{E}:=\{\dot{u} \mid u \in E\}
$$


An ordered pair $(\mathfrak{P}(U), \mathfrak{B})$ is alleged to be a hyper-measurable structure on $U$ if $\mathfrak{B}$ could be a $\sigma$-field in $\mathfrak{P}(U)$ and $\dot{U} \subseteq \mathfrak{B}$. Given a probability space $(\Omega, \mathfrak{A}, P)$ and a hyper-measurable structure $(\mathfrak{P}(U), \mathfrak{B})$ on $U$, a random set on $U$ is outlined to be a mapping $\xi: \Omega \rightarrow \mathfrak{P}(U)$ that is $\mathfrak{A}-\mathfrak{B}$ measurable, that is,

$$
(\forall C \in \mathfrak{B})\left(\xi^{-1}(C):=\{\omega \mid \omega \in \Omega \text { and } \xi(\omega) \in C\} \in \mathfrak{A}\right) .
$$

Suppose that $\xi$ is a random set on $U$. Let

$$
\tilde{H}(u):=P(\omega \mid u \in \xi(\omega)) \text { for each } u \in U .
$$

Then $\tilde{H}$ is a kind of fuzzy set in $U$. We tend to decision $\tilde{H}$, a falling shadow of the random set $\xi$, and $\xi$ is termed a cloud of $\tilde{H}$.

For example, $(\Omega, \mathfrak{A}, P)=([0,1], \mathfrak{A}, m)$, wherever $\mathfrak{A}$ could be a Borel field on $[0,1]$ and $m$ is the usual Lebesgue measure. Let $\tilde{H}$ be a fuzzy set in $U$ and $\tilde{H}_{t}:=$ $\{u \in U \mid \tilde{H}(u) \geq t\}$ be a t-cut of $\tilde{H}$. Then $\xi:[0,1] \rightarrow \mathfrak{P}(U), t \mapsto \tilde{H}_{t}$ is a random set and $\xi$ is a cloud of $\tilde{H}$. The decision $\xi$ outlined on top is cut-cloud of $\tilde{H}$.

\section{Falling Fuzzy Implicative Filters}

In this section, we introduce the thought of falling fuzzy implicative filter of a BEalgebra and study its properties. In what follows, let $X$ denote a BE-algebra unless otherwise given.

Definition 3.1. $\quad$ [1] Let $(\Omega, \mathfrak{A}, P)$ be a probability space and

$$
\xi: \Omega \rightarrow \mathfrak{P}(X)
$$

a random set. If $\xi(\omega)$ is a filter of $X$ for any $\omega \in \Omega$, then the falling shadow $\tilde{H}$ of the random set $\xi$, i.e.,

$$
\tilde{H}(x)=P(\omega \mid x \in \xi(\omega))
$$

is called a falling fuzzy filter of $X$.

Let $(\Omega, \mathfrak{A}, P)$ be a probability space and let

$$
F(X):=\{f \mid f: \Omega \rightarrow X \text { is a mapping }\}
$$

where $X$ is a BE-algebra. Define an operation $\otimes$ on $F(X)$ by

$$
(\forall \omega \in \Omega)((f \otimes g)(\omega)=f(\omega) * g(\omega))
$$

for all $f, g \in F(X)$. Let $\theta \in F(X)$ be defined by $\theta(\omega)=1$ for all $\omega \in \Omega$. Then it can be easily verified that $(F(X), \otimes, \theta)$ is a BE-algebra.

Definition 3.2. Let $(\Omega, \mathfrak{A}, P)$ be a probability space and

$$
\xi: \Omega \rightarrow \mathfrak{P}(X)
$$


a random set. If $\xi(\omega)$ is an implicative filter of $X$ for any $\omega \in \Omega$, then the falling shadow $\tilde{H}$ of the random set $\xi$, i.e.,

$$
\tilde{H}(x)=P(\omega \mid x \in \xi(\omega))
$$

is called a falling fuzzy implicative filter of $X$.

For any subset $A$ of $X$ and $f \in F(X)$, let

$$
\begin{gathered}
A_{f}:=\{\omega \in \Omega \mid f(\omega) \in A\}, \\
\xi: \Omega \rightarrow \mathfrak{P}(F(X)), \omega \mapsto\{f \in F(X) \mid f(\omega) \in A\} .
\end{gathered}
$$

Then $A_{f} \in \mathfrak{A}$.

Theorem 3.1. If $F$ is an implicative filter of $X$, then

$$
\xi(\omega)=\{f \in F(X) \mid f(\omega) \in F\}
$$

is an implicative filter of $F(X)$.

Proof. Assume that $F$ is an implicative filter of $X$ and let $\omega \in \Omega$. Since $\theta(\omega)=1 \in F$, we have $\theta \in \xi(\omega)$. Let $f, g, h \in F(X))$ be such that $f \otimes g \in \xi(\omega)$ and $f \otimes(g \otimes h) \in$ $\xi(\omega)$. Then $f(\omega) *(g(\omega) * h(\omega))=f(\omega) *((g \otimes h)(\omega))=(f \otimes(g \otimes h)(\omega) \in F$ and $f(\omega) * g(\omega) \in F$. Since $F$ is an implicative filter of $X$, we have $(f \otimes h)(\omega)=$ $f(\omega) * h(\omega) \in F$ and so $f \otimes h \in \xi(\omega)$. Hence $\xi(\omega)$ is an implicative filter of $F(X)$.

Since $\xi^{-1}(\dot{f})=\{\omega \in \Omega \mid f \in \xi(\omega)\}=\{\omega \in \Omega \mid f(\omega) \in A\}=A_{f} \in \mathfrak{A}$, we see that $\xi$ is a random set on $F(X)$. Let $\tilde{H}(f)=P(\omega \mid f(\omega) \in A)$. Then $\tilde{H}$ is a falling fuzzy implicative filter of $F(X)$.

Example 3.1. Consider the set $X=\{1, a, b, c\}$ with the following table of $*$ :

\begin{tabular}{|c|c|c|c|c|}
\hline$*$ & 1 & $\mathrm{a}$ & $\mathrm{b}$ & $\mathrm{c}$ \\
\hline 1 & 1 & $\mathrm{a}$ & $\mathrm{b}$ & $\mathrm{c}$ \\
\hline $\mathrm{a}$ & 1 & 1 & $\mathrm{~b}$ & $\mathrm{c}$ \\
\hline $\mathrm{b}$ & 1 & $\mathrm{a}$ & 1 & $\mathrm{c}$ \\
\hline $\mathrm{c}$ & 1 & 1 & 1 & 1 \\
\hline
\end{tabular}

Then $(X, *, 1)$ is a BE-algebra. Let $(\Omega, \mathfrak{A}, P)=([0,1], \mathfrak{A}, m)$ and $\xi:[0,1] \rightarrow \mathfrak{P}(X)$ be defined by

$$
\xi(t)= \begin{cases}\{1, a\}, & \text { if } t \in[0,0.4) \\ \{1, b\}, & \text { if } t \in[0.4,1] .\end{cases}
$$

Then $\xi(t)$ is an implicative filter of $X$ for all $t \in[0,1]$. Hence $\tilde{H}(x)=P(t \mid x \in \xi(t))$ is a falling fuzzy implicative filter of $X$ and $\tilde{H}$ is represented as follows

$$
\tilde{H}(x)= \begin{cases}1, & \text { if } \mathrm{x}=1 \\ 0.4, & \text { if } \mathrm{x}=\mathrm{a} \\ 0.6, & \text { if } \mathrm{x}=\mathrm{b} \\ 0, & \text { if } \mathrm{x}=\mathrm{c}\end{cases}
$$

In this case, we can easily check that $\tilde{H}$ is a fuzzy implicative filter of $X$. 
Example 3.2. Consider the set $X=\{1, a, b, c\}$ with the following table of $*$ :

\begin{tabular}{|c|c|c|c|c|}
\hline$*$ & 1 & $\mathrm{a}$ & $\mathrm{b}$ & $\mathrm{c}$ \\
\hline 1 & 1 & $\mathrm{a}$ & $\mathrm{b}$ & $\mathrm{c}$ \\
\hline $\mathrm{a}$ & 1 & 1 & $\mathrm{~b}$ & $\mathrm{~b}$ \\
\hline $\mathrm{b}$ & 1 & $\mathrm{a}$ & 1 & $\mathrm{a}$ \\
\hline $\mathrm{c}$ & 1 & 1 & 1 & 1 \\
\hline
\end{tabular}

Then $(X, *, 1)$ is a BE-algebra. Let $(\Omega, \mathfrak{A}, P)=([0,1], \mathfrak{A}, m)$ and $\xi:[0,1] \rightarrow \mathfrak{P}(X)$ be defined by

$$
\xi(t)= \begin{cases}\{1, a\}, & \text { if } t \in[0,0.6) \\ \{1, b\}, & \text { if } t \in[0.6,1] .\end{cases}
$$

Then $\xi(t)$ is an implicative filter of $X$ for all $t \in[0,1]$. Hence $\tilde{H}(x)=P(t \mid x \in \xi(t))$ is a falling fuzzy implicative filter of $X$ and $\tilde{H}$ is represented as follows

$$
\tilde{H}(x)= \begin{cases}1, & \text { if } \mathrm{x}=1 \\ 0.4, & \text { if } \mathrm{x}=\mathrm{b} \\ 0.6, & \text { if } \mathrm{x}=\mathrm{a} \\ 0, & \text { if } \mathrm{x}=\mathrm{c}\end{cases}
$$

But $\tilde{H}$ is not a fuzzy implicative filter of $X$. Since $\tilde{H}(1 * c)=\tilde{H}(c)=0<0.4=$ $\min \{\tilde{H}(b), \tilde{H}(a)\}=\min \{\tilde{H}(a * c), \tilde{H}(a)\}=\min \{\tilde{H}(1 *(a * c)), \tilde{H}(1 * a)\}$

Theorem 3.2. Every fuzzy implicative filter of $X$ is a falling fuzzy implicative filter of $X$.

Proof. Let $\tilde{H}$ be a fuzzy implicative filter of $X$. Then $\tilde{H}_{t}$ is an implicative filter of $X$ for all $t \in[0,1]$. Let $\xi:[0,1] \rightarrow \mathfrak{P}(X)$ be a random set and $\xi(t)=\tilde{H}_{t}$. Then $\tilde{H}$ is a falling fuzzy implicative filter of $X$.

Example 3.2 shows that the converse of the above theorem is not valid.

Theorem 3.3. Every falling fuzzy implicative filter of $X$ is a fuzzy filter of $X$.

Proof. Let $\tilde{H}$ be a falling fuzzy implicative filter of $X$. Then $\xi(\omega)$ is an implicative filter of $X$ and hence it is a filter of $X$. Therefore, $\tilde{H}$ is a falling fuzzy filter of $X$.

The converse of the above theorem need not be true as shown by the subsequent example.

Example 3.3. Consider the set $X=\{1, a, b, c, d, e\}$ with the following table of $*$ :

\begin{tabular}{|c|c|c|c|c|c|c|}
\hline$*$ & 1 & $\mathrm{a}$ & $\mathrm{b}$ & $\mathrm{c}$ & $\mathrm{d}$ & $\mathrm{e}$ \\
\hline 1 & 1 & $\mathrm{a}$ & $\mathrm{b}$ & $\mathrm{c}$ & $\mathrm{d}$ & $\mathrm{e}$ \\
\hline $\mathrm{a}$ & 1 & 1 & $\mathrm{a}$ & $\mathrm{c}$ & $\mathrm{c}$ & $\mathrm{d}$ \\
\hline $\mathrm{b}$ & 1 & 1 & 1 & $\mathrm{c}$ & $\mathrm{c}$ & $\mathrm{c}$ \\
\hline $\mathrm{c}$ & 1 & $\mathrm{a}$ & $\mathrm{b}$ & 1 & $\mathrm{a}$ & $\mathrm{b}$ \\
\hline $\mathrm{d}$ & 1 & 1 & $\mathrm{a}$ & 1 & 1 & $\mathrm{a}$ \\
\hline $\mathrm{e}$ & 1 & 1 & 1 & 1 & 1 & 1 \\
\hline
\end{tabular}


Then $(X, *, 1)$ is a BE-algebra. Let $(\Omega, \mathfrak{A}, P)=([0,1], \mathfrak{A}, m)$ and $\xi:[0,1] \rightarrow \mathfrak{P}(X)$ be defined by

$$
\xi(t)= \begin{cases}\{1, a\}, & \text { if } t \in[0,0.2) \\ \{1, c\}, & \text { if } t \in[0.2,0.7) \\ \{1, a, b\}, & \text { if } t \in[0.7,1] .\end{cases}
$$

Then $\xi(t)$ is a filter of $X$ for all $t \in[0,1]$. Hence $\tilde{H}(x)=P(t \mid x \in \xi(t))$ is a falling fuzzy filter of $X$ and $\tilde{H}$ is represented as follows

$$
\tilde{H}(x)= \begin{cases}1, & \text { if } \mathrm{x}=1 \\ 0.5, & \text { if } \mathrm{x}=\mathrm{a}, \mathrm{c} \\ 0.3, & \text { if } \mathrm{x}=\mathrm{b} \\ 0, & \text { if } \mathrm{x}=\mathrm{d}, \mathrm{e}\end{cases}
$$

If $t \in[0.2,0.7)$, then $\xi(t)=\{1, c\}$ which is not an implicative filter of $X$. Since $a *(d * e)=$ $a * a=1 \in \xi(t)$ and $a * d=c \in \xi(t)$, but $a * e=d \notin \xi(t)$. Therefore $\tilde{H}$ is not a falling fuzzy implicative filter of $X$.

Let $X$ be a BE-algebra and $(\Omega, \mathfrak{A}, P)$ a probability space. Let $\tilde{H}$ be a falling shadow of a random set $\xi: \Omega \rightarrow \mathfrak{P}(X)$. For $x \in X$, let

$$
\Omega(x ; \xi)=\{\omega \in \Omega \mid x \in \xi(\omega)\} .
$$

Then $\Omega(x ; \xi) \in \mathfrak{A}$.

Theorem 3.4. [1] If $\tilde{H}$ is a falling fuzzy filter of $X$, then for all $x, y \in X$ :

(1) $x \leq y \Rightarrow \Omega(x ; \xi) \subseteq \Omega(y ; \xi)$

(2) $\Omega(x * y ; \xi) \cap \Omega(x: \xi) \subseteq \Omega(y ; \xi)$

(3) $\Omega(x ; \xi) \subseteq \Omega(1 ; \xi)$

(4) $\Omega(y ; \xi) \subseteq \Omega(x * y ; \xi)$.

Theorem 3.5. If a falling shadow $\tilde{H}$ of a random set $\xi: \Omega \rightarrow \mathfrak{P}(X)$ is a falling fuzzy implicative filter of $X$, then:

(i) $(\forall x, y, z \in X) \quad(\Omega(x *(y * z) ; \xi) \cap \Omega(x * y ; \xi) \subseteq \Omega(x * z ; \xi)$.

(ii) $(\forall x, y, z \in X) \quad(\Omega(x * z ; \xi) \subseteq \Omega(x *(y * z) ; \xi))$.

Proof. (i) Let $\omega \in \Omega(x *(y * z) ; \xi) \cap \Omega(x * y ; \xi)$. Then $x *(y * z) \in \xi(\omega)$ and $x * y \in \xi(\omega)$.. Since $\xi(\omega)$ is an implicative filter of $X$, we have from $(F 3)$ that $x * z \in \xi(\omega)$ and so $\omega \in \Omega(x * z ; \xi)$. Therefore

$$
\Omega(x *(y * z) ; \xi) \cap \Omega(x * y ; \xi) \subseteq \Omega(x * z ; \xi)
$$

for all $x, y, z \in X$.

(ii) Since $x * z \leq x *(y * z)$, we have from Theorem 3.4(1) that

$$
\Omega(x * z ; \xi) \subseteq \Omega(x *(y * z) ; \xi) .
$$


Finally, we conclude this section with the following theorem that shows that every falling fuzzy implicative filter of $X$ could be a $T_{m}$-fuzzy implicative filter of $X$.

Theorem 3.6. If $\tilde{H}$ is a falling fuzzy implicative filter of a BE-algebra $X$ then for all $x, y \in X$,

$$
\tilde{H}(x * z) \geq T_{m}(\tilde{H}(x *(y * z)), \tilde{H}(x * y))
$$

where $T_{m}(s, t)=\max \{s+t-1,0\}$ for any $s, t \in[0,1]$.

Proof. By definition, $\xi(\omega)$ is an implicative filter of $X$ for any $\omega \in \Omega$. Hence

$$
\{\omega \in \Omega \mid x *(y * z) \in \xi(\omega)\} \cap\{\omega \in \Omega \mid x * y \in \xi(\omega)\} \subseteq\{\omega \in \Omega \mid x * z \in \xi(\omega)\}
$$

and thus

$$
\begin{aligned}
\tilde{H}(x * z)= & P(\omega \mid x * z \in \xi(\omega)) \\
\geq & P(\{\omega \mid x *(y * z) \in \xi(\omega)\} \cap\{\omega \mid x * y \in \xi(\omega)\}) \\
\geq & P(\omega \mid x *(y * z) \in \xi(\omega))+P(\omega \mid x * y \in \xi(\omega)) \\
& -P(\omega \mid x *(y * z) \in \xi(\omega) \text { or } \omega \mid x * y \in \xi(\omega)) \\
\geq & \tilde{H}(x *(y * z))+\tilde{H}(x * y)-1
\end{aligned}
$$

Hence $\tilde{H}(x * z) \geq \max \{\tilde{H}(x *(y * z))+\tilde{H}(x * y)-1,0\}=T_{m}(\tilde{H}(x *(y * z)), \tilde{H}(x * y))$.

\section{Conclusion}

The falling shadow illustration theory shows us the way of choice relaid on the joint degrees distributions. It is a reasonable and convenient approach for theoretical development and allows for sensible applications of fuzzy sets and fuzzy logics. The speculation on falling shadows relates likelihood ideas to the membership functions of fuzzy sets. In this paper, we established a theoretical approach for outlining a fuzzy implicative filter in an exceedingly BE-algebra supported by the speculation of falling shadows. We provided relations between fuzzy implicative filters and falling fuzzy implicative filters and showed that every falling fuzzy implicative filter could be a $T_{m}$-fuzzy implicative filter. Based on these results, we plan to apply in our future study the speculation of falling shadows to the opposite varieties of filters in BE-algebras.

\section{REF EREN C ES}

1. R.K. Bandaru, N.Rafi and B. Davvaz: Falling Fuzzy filters in BE-algebras, Journal of the Chungcheong Mathematical Society, 30(2) (2017), 201-211.

2. G. Dymek, A. Walendziak: Fuzzy Filters of BE-algebras. Math.Slovaca, 63(5) (2013), 935-946.

3. I.R. Goodman: Fuzzy sets as equivalent classes of random sets. In Recent Developments in Fuzzy Sets and Possibility Theory(R.Yager, ed.), Pergamon Press, New York, 1982. 
4. Y.B.Jun and M.S.Kang: Fuzzy positive implicative ideals of BCK-algebras based on the theory of falling shadows. Computers and Mathematics with Applications, 61(1) (2011), 62-67.

5. Y.B.Jun and C.H.Park: Falling shadows applied to subalgebras and ideals of BCK/BCI-algebras. Honam Mathematical J. 34(2) (2012), 135-144.

6. H.S.Kim and Y.H.Kim: On BE-algebras. Sci.Math.Jpn. 66(1) (2007), 113-116.

7. M.S. Rao: Fuzzy implicative filters of BE-algebras. Annals of Fuzzy Mathematics and Informatics, 6(3) (2013), 755-765.

8. S.Shin Ahn, Y.H.Kim and K. Sook So: Fuzzy BE-algebras. J.Appl.Math. \& Informatics, 29(3-4) (2011), 1049-1057.

9. S.K.Tan, P.Z.Wang and X.Z.Zhang: Fuzzy inference relation based on the theory of falling shadows. Fuzzy Sets and Systems, 53 (1993), 179-188.

10. S.K.Tan, P.Z.Wang and E.S.Lee: Fuzzy set operations based on the theory of falling shadows. J.Math.Anal.Appl. 174 (1993), 242-255.

11. P.Z.Wang: Fuzzy Sets and Falling Shadows of Random Sets Beijing Normal University Press, China, 1985(in Chinese).

12. P.Z.Wang and E.Sanchez: Treating a fuzzy subset as a projectable random set, in Fuzzy Information and Decision. M.M.Gupta, E. Sanchez(eds.), Pergamon Press, 1982, pp. 212-219.

13. J.Zhan, D.Pci and Y.B.Jun: Falling fuzzy (implicative) filters of $R_{0}-$ algebras and its applications. J.Intell.Fuzzy Systems, 24 (2013), 611-618.

\section{Ravi Kumar Bandaru}

Department of Mathematics

GITAM School of Technology

GITAM Hyderabad

Telangana-502329

India

ravimaths83@gmail.com

N. Rafi

Department of Mathematics

Bapatla Engineering College

Bapatla

Andhra Pradesh-522 101

India rafimaths@gmail.com

Bijan Davvaz

Department of Mathematics

Yazd University

Yazd, Iran

davvaz@yazd.ac.ir 